Anti-complementary Readings.-Forty-two tests were performed on twenty-one cases. In cases to which both the Kline test and the Wassermann reaction had been applied initially, and in which the Wassermann result had been anti-complementary, repetition of the Wassermann yielded a result corresponding to the original Kline result in 89 per cent. of the cases. These results are in accordance with the views put forward by $\mathrm{Miller}^{3}$ and Stewart. ${ }^{4}$ We also agree with the latter when he says that the responsibility for a false positive Kline test cannot be attributed to the substance responsible for the anti-complementary Wassermann reading.

\section{Discussion and Summary}

An outstanding feature is the absolute agreement of the Wassermann reaction and the Kline test in the untreated syphilitic cases in Table I. We are of the opinion that the Kline test has a definite place in the diagnosis of syphilis, and that on the basis of sensitivity and selectivity it constitutes a suitable adjuvant to, but an indifferent substitute for, a universal test such as the Wassermann. It is relatively simple, ${ }^{5}$ rapid, and inexpensive, while only a very small quantity of serum is necessary for its performance; but despite these advantages it seems unsuitable for use save in laboratories (where large numbers of sera are presented for examination at one time), since the antigen emulsion-an expensive itemonly retains its efficiency for a relatively short period of time. In addition, considerable practice is essential before minor deviations from the normal can be accurately assessed.

We have already emphasized the specific sensitivity of the test only in that it remains longer positive in the later stages of syphilis under treatment, and in that respect we agree with Kilduffe, ${ }^{6}$ who compared his results with the Kolmer modification of the Wassermann-essentially a very delicate test. Our findings also coincided with those of Gray, ${ }^{7}$ who found it of value in assessing the result of antispecific treatment. Osmond and Hughes ${ }^{8}$ remark that it is predominantly an eliminating test rather than a diagnostic one, and with these views we are in accord. They suggest, also, that it be used as a serological index in testing for cure. Allusion has already been made to the use of the Kline test where the Wassermann has given an anti-complementary result.

We might add that the test was repeated in all our cases which showed disagreement with the Wassermann, since our experience was similar to that of Kilduffe in regard to the possibility of results occasionally varying with the use of different serological tests.

We hold, therefore, that the Kline has its chief value: (1) in the later stages of syphilis under treatment; (2) in cases where it is especially important that syphilis should be excluded-for example, interstitial keratitis; and (3) as a general supplement to the Wassermann reaction.

Our best thanks are due to Drs. Logan, Batchelor, and Smeall for their valuable advice, and to Dr. Elder for kindly allowing us to include certain cases of tuberculosis in our series.

\section{References}

${ }^{1}$ Kline, B. S., and Littman, S.: Amer. Journ. Syph., 1931, xv, 405. 'Chargin, L., and Rosenthal, T.: Journ. Lab. and Clin. Med., 1930, xv, 1008.

${ }^{3}$ Miller, T. H.: Amer. Journ. Syph., 1929, xiii, 583

4 Stewart, H. V.: Ibid., 1931, xv, 234

5 Ashworth, P. G., and Irving. D. H.: Med. Journ. of Australia, $1932, \mathrm{i}, 14$.

Gildufie, R. A.: Amer. Journ. Clin. Path., 1931, i, 69

7 Gray, J. D. Allan: Edinburgh Med. Journ., 1933, xl, 305.

${ }^{8}$ Osmond, T. E., and Hughes K. E.: Lancet, 1932, i, 130.

\section{ABDOMINAL HYDROCELE WITH RECORD OF TWO CASES}

BY

A. D. CHARTERS, M.D.CAMb., D.T.M. AND H.LOND.

RESIDENT MEDICAL OFFICER TO THE NAKLRU WAR MEMIORIAL HOSPTTAL, KENYA ; FORMERLY SURGEON TO THE C.M.S. HOSPITAL, NGORA, CGANDA

On perusing the literature I can only find reference to thirty-six previously recorded cases of this condition, which is therefore of sufficient rarity to warrant publication of my cases, particularly in view of the prominence lent to the disease by the recent appearance of two other published accounts.

Case I

The patient, a tall, thin man, aged 30 , of the Buganda tribe, was admitted to the C.M.S. Hospital, Ngora, Uganda, on October 29th, 1930. He gave a five-years history of a swelling which started in the upper part of the right side of his scrotum, gradually increased in size, and spread to his abdomen.

On examination the right side of his scrotum was distended by a large hydrocele, which was evidently continuous with a large abdominal tumour. The latter resembled in appearance an eight months pregnant uterus, but could be seen to be lobulated into two parts by a constriction below the umbilicus. When the patient was in the erect position the tumour descended and became more prominent, while the scrotal swelling increased in size. On palpation the abdominal swelling was found to be tense and cystic, and to have a rounded outline. It had a well-defined edge, and was freely movable within the abdomen. Both fluctuation and fluid thrill could be elicited between different parts of the tumour and between the abdomen and scrotum, and fluid could be displaced from scrotum to abdomen and vice versa. Percussion revealed dullness over the tumour and resonance in the flanks. The size of the mass can be appreciated by a study of its boundaries within the abdomen. When pressure was applied over the lower part of the scrotum the upper limit of the abdominal tumour was just overlapped by the costal margin, but when this pressure was relaxed its superior border receded to a point 1 inch below that margin. Its upper limit on the right side was not so high, being only 1 inch above the umbilicus. The tumour extended on the left side to a point 2 inches to the outer side of the left anterior superior iliac spine, and for a corresponding distance into the right flank. There was no tenderness. The right side of the scrotum had the usual tense elastic characters of a hydrocele, but these features became more marked when pressure was made upon the abdomen. The hydrocele was of large size, extending for a distance of 11 inches below the right pubic spine, and the scrotum, whose girth at its widest level measured 19 inches, contained on the left side a slightly smaller hydrocele, which had no communication with the abdomen. A diagnosis of abdominal hydrocele was made and operation advised.

An operation was performed on November 4th, an incision 4 inches long, with its upper extremity at the level of the pubic spine, was made over the upper part of the scrotum on the right side. The tunica vaginalis was exposed and separated from its scrotal coverings with the finger. It was pierced with trocar and cannula, and four pints of strawcoloured fluid were removed. The evacuation of the fluid was accompanied by collapse of the abdominal tumour. After enlarging the aperture in the tunica vaginalis it was possible to pass a finger into the abdominal loculus, but the limits of the hydrocele could not be reached. By means of gauze dissection combined with traction from below, the whole sac was drawn down from the abdomen. It was surprising how easily this separation could be carried out. In the abdominal portion was a completely isolated loculus about the size of a golf ball, which contained fluid of the same yellow colour. The whole hydrocele sac was excised, with the exception of a small portion sufficient to evert and suture with catgut behind the cord. A rubber drainage tube was inserted into the upper end of the wound, and removed after forty-eight hours. A high scrotal incision, 3 inches long, 
was then made on the left side. Nineteen ounces of strawcoloured fluid were drawn off, and the hydrocele was cured by excision of the redundant sac and by suture of the remainder in eversion behind the cord. A drainage tube was retained for forty-eight hours. There was no communication with the abdomen on this side. As soon as the patient began to come round from the anaesthetic and gave a cough, a large right inguinal hernia appeared. $\mathrm{He}$ was discharged on December 13th with complete cure of hydroceles on both sides, and with no trace of hernia. It is evident that the raw surface left after separation of the sac resulted in adhesions, which effectively closed the hernial aperture.

\section{CASE II}

The patient, a man aged about 30 , of the Alango tribe, was admitted to the C.M.S. Hospital, Ngora, on June 6th, 1931, with a history of swelling of the right side of his scrotum for three years and of the left side for two years. On examination there was a large hydrocele on each side of the scrotum, whose circumference measured 16 inches, the lower limit of the scrotum being at a distance of 12 inches from the pubic spine.

An operation was carried out on June 9th. Radical cure was performed by means of excision of the redundant sac and suture of the remainder in eversion through a high scrotal incision on each side. The hydrocele on the right side contained $2 \frac{1}{2}$ pints of fluid, and the sac was found to extend upwards into the inguinal canal, from which it was separated by traction combined with gauze dissection. On the left side the hydrocele contained 16 ounces of fluid, and, although the sac did not extend into the inguinal canal, the upper part was completely isolated from the remainder as a separate loculus. Immediately after the operation a right inguinal hernia appeared. It is therefore probable that there was on the right side a small protrusion of the sac into the abdominal cavity. When the patient was discharged on July 2nd with cure of both hydroceles it was found that the hernia had undergone spontaneous cure.

\section{Causation}

Several theories have been propounded to explain the formation of abdominal hydrocele. Macewen's views ${ }^{1}$ can best be summarized by quoting his own words:

"The sac being of peritoneal origin, one ought to consider the possibility of the funiculo-vaginal portion of the peritoneum, instead of terminating on the abdominal side at the internal ring, being carried as a sheath along the anterior aspect of the pelvic portion of the cord, so that this funiculovaginal process of peritoneum would be continuous and patent from the testicular covering to the deepest part of the pelvic portion of the cord. When this elongated sac became distended with fluid, a bilocular hydrocele would form, the constriction being made by the aperture in the abdominal muscles through which it passed."

Firth $^{2}$ has pointed out that, if this theory were correct, the peritoneum would be raised in front of the sac, and an incision through the abdominal wall would have to divide the peritoneum twice before exposing the sac of the hydrocele. Such a relationship of peritoneum to sac was actually observed by both Coleman ${ }^{3}$ and Lasbrey. ${ }^{4}$ In Tillmans's case, however, the cyst lay between the parietes and peritoneum.

The most probable explanation, and one which has been supported by Jacobson, Reclus, and Villeneuve, is that distension with fluid occurs in a funiculo-vaginal process which is patent from the scrotum up to the internal abdominal ring. The internal pressure within such a sac causes expansion into the abdomen. Case II was probably a very early stage of this process.

This theory is supported by the following observations: (1) In both of my cases the disease was first noticed in the scrotum, which would at first offer little resistance to expansion. (2) In both cases the scrotal hydrocele was distended to a large size, and was complicated by a hydrocele on the opposite side, which would limit further expansion of the scrotum. (3) The history was of long duration in both cases-five years in Case I and three years in Case II. It would take a considerable length of time to cause sufficient distension of the lower sac to allow of formation of an abdominal loculus by expansion against the intra-abdominal pressure. Moreover, a reference to the literature of the last thirty years revealed that in every published account in which a description of the history could be found the condition had been present for over two years, with the exception of a case described by Richards, ${ }^{6}$ who pointed out that the history was unreliable, especially as regards dates. Further, Lakhoti, ${ }^{7}$ Coleman, Lasbrey, and Richards all state in their respective papers that the condition was first noticed in the scrotum, with subsequent involvement of the abdomen. It should be admitted that Macewen opposed this theory, on the assumption that the abdominal pressure would make such a course of events unlikely.

Firth drew attention to the congenital peritoneal pouches near the internal abdominal ring, which were described by Rokitansky and regarded by Moynihan as resulting from an aberrant detachment of the gubernaculum to the peritoneum, and believes that in a similar way the gubernaculum may be concerned in the development of bilocular hydroceles. It may be recalled that Moynihan ${ }^{5}$ drew a close anatomical and developmental analogy between properitoneal hernia and bilocular hydrocele, and it is on the assumption of this relationship that the varying position of the upper sac of this form of hydrocele is explained.

\section{Differential Diagnosis}

In the advanced stage, exemplified by Case $I$, the diagnosis should offer little difficulty, provided that the scrotum is examined in every case of cystic abdominal tumour in the male. Lewtas, ${ }^{8}$ however, pointed out that an early abdominal loculus may be mistaken for an inguinal hernia. The diagnosis rests upon the dullness on percussion of the swelling in the groin, its re-descent without gurgling after reduction, the translucency of the external swelling, and the presence of an area of dullness extending inwards or upwards from the internal ring. The dark pigmentation of his skin renders the test of transillumination of no value in the African native. It is very easy to miss an early abdominal loculus before operation in the examination of such a patient as Case II, but it should be possible to determine its presence at operation if a finger is passed into the upper part of the sac to define its limits before separation.

\section{Treatment}

In the textbooks which I have consulted I can find no mention of the treatment. From the variety of operation performed in the past there appears to be no settled method of dealing with the condition. Tapping is merely palliative; injection is dangerous. Drainage which was performed by both Lewtas and Bickle, is liable to result in recurrence. Excision of the sac is evidently the method of choice. I have already noted the remarkable ease with which separation of the abdominal sac in Case I was performed. Nearly all the surgeons who have practised this method of treatment have made similar observations. Coleman and Lasbrey, on the other hand, both considered it necessary, after separating the sac as far as possible through a scrotal incision without withdrawing the fluid, to make a second incision in the anterior abdominal wall to facilitate the 
removal. It is probable that, had the fluid been allowed to escape, removal of the sac through the scrotal route would have proved quite a simple matter without need of a second incision. Firth, Richards, and Hermann ${ }^{10}$ all excised the sac through an inguinal incision opening the inguinal canal, and each surgeon, after taking away the sac, obliterated the inguinal canal by their various methods of choice.

The operation which I recommend has been already outlined in the description of my cases. No plastic repair of the inguinal canal was performed, and the hernias which resulted when the patients strained immediately after the operation underwent spontaneous cure. It is therefore clear that in some cases, possibly in all cases which have been submitted to the correct after-treatment, no subsequent operation for hernia is needed.

It should be borne in mind by those surgeons who favour repair of the inguinal canal that, although the internal abdominal ring and inguinal canal have been dilated, the condition after removal of a hydrocele sac differs in the following respects from that after excision of the sac of an inguinal hernia.

1. In the former there is no stump of hernial sac and there has been no dragging down of the parietal peritoneum; on the contrary the reverse is the case, for the parietal peritoneum, by being pushed up and compressed, has probably become reduced in area in order to accommodate itself to the altered conditions.

2. After the radical cure of an inguinal hernia there is frequently increased intra-abdominal pressure, whereas after extirpation of a bilocular hydrocele this pressure is always reduced in proportion to the size of the abdominal loculus removed.

3. After separation of the sac from the peritoneum on the one side and from the abdominal parietes on the other, raw surfaces are left which result in adhesion of the parietal peritoneum in the region of the internal abdominal ring, and for a distance around this area proportional to the sac which was withdrawn.

After radical operation upon the enormous hernias which are quite common in the natives of the Teso district of Uganda, it is always a difficult problem to prevent the accumulation of blood in the bulky, lax, and pendulous scrotums which remain. For this reason, in those cases where an inguinal hernia and a large hydrocele coexist in the same patient, I never treat the two conditions at the same operation. Incidentally, I prefer to operate on the hernia first, because the distension of the scrotum by the hydrocele effectually prevents any accumulation of blood in this region. I therefore suggest that the treatment of bilocular hydrocele should consist of separation and removal of the whole of the abdominal and of the redundant scrotal portion of the tunica vaginalis, with suture of the remainder in eversion behind the cord, through a high scrotal incision, followed by drainage by means of a rubber tube for forty-eight hours. The patient should then be maintained.in the recumbent position for three weeks in order to allow firm adhesions to develop and prevent hernia formation. No attempt should be made to operate on the hernia, which will probably disappear, but which, if persistent, will be more satisfactorily treated at a later date.

\section{REFERENCES}

Macewen, W.: Practitioner, 1896, lvii, 123

2 Firth, J. W.: British Medical Journal, 1901, ii, 1463.

3 Coleman, R. B.: Ibid., 1918, ii, 629.

4 Lasbrey, F. O.: Ibid., 1916, ii, 292.

s Moynihan, B. G. A.: Lancet, 1900, i, 516

- Richards, O.: Ibid., 1908, ii, 533.

TRichards, O.: Ibid., 1908, ii, 533.

'Lakhoti, B.: Indian Med. Gaz.,

8 Lewtas, J.: Lancet, 1905, i, 489.

- Bickle, L. W.: British Medical Journal, 1919, ii, 13

10 Hermann, S. F.: Journ. Amer. Med. Assoc., 1932, xcviii, 399.

\section{Clinical Memoranda}

\section{Inversion of the Temperature in Acute Lobar Pneumonia}

During the course of an investigation of over one hundred cases of acute lobar pneumonia at Smithdown Road Hospital, Liverpool, the temperature in one patient was found to assume an inverse type. The morning temperatures were higher than those of the previous evenings, and the symptoms were more severe during the day. The crisis also was diurnal. This is very unusual in acute febrile conditions, for in such, as a rule, the evening temperature is higher than that of the morning. Moreover, the symptoms are more severe during the night, at which time also the crisis occurs.

The case in question was that of a night watchman, aged 39. On November $3 \mathrm{rd}, 1933$, he was seized with acute pain in the left chest, accompanied by cough and vomiting, and was admitted to hospital the following day. Clinical examination revealed some consolidation of the left lower lobe. The whole of the left lung eventually became consolidated. The following was his temperature chart.

\begin{tabular}{|c|c|c|c|c|}
\hline \multicolumn{3}{|c|}{ Date } & Morning & Evening \\
\hline Nov. 4 th, 193 & $\ldots$ & $\ldots$ & - & $100.0^{\circ}$ \\
\hline " 5th, . & $\ldots$ & ... & $101.0^{\circ}$ & $97.0^{\circ}$ \\
\hline .6 th, & $\ldots$ & $\ldots$ & $101.2^{\circ}$ & $99.6^{\circ}$ \\
\hline$\|$ 7th, & $\ldots$ & $\ldots$ & $100.0^{\circ}$ & $97.8^{\circ}$ \\
\hline$"$ 8th, & $\ldots$ & $\ldots$ & $102.8^{\circ}$ & Sleeping \\
\hline " 9th, & $\ldots$ & ... & $99.6^{\circ}$ & $99.0^{\circ}$ \\
\hline , 10th, & $\ldots$ & $\ldots$ & $98.4^{\circ}$ & $97.0^{\circ}$ \\
\hline
\end{tabular}

\section{Two Other Cases from the Literature}

There appear to be only two similar cases on record. The first was that quoted by Dr. Downing in the British Medical Journal of July 1st, 1911. He stated:

"Possibly in night workers the temperature chart is upset in this way. It would be interesting to know if practitioners in attendance on men whose employment takes them on duty at night have observed a similar phenomenon."

In reply to this Dr. Hingston ${ }^{1}$ quoted a case occurring in his practice, and added:

"That an 'inverse' occupation-namely, night work and day sleep-should produce an inverse type of temperature in disease is a feasible solution of the temperature phenomenon, since it is well known that in such people during health the temperature also tends to become inverse. So, also, since the crisis in pneumonia seems usually to occur at a time when the symptoms are most trying and the temperature is highest, it might, in night workers, be expected to occur during the morning hours when the fever has reached its maximum, rather than during the evening hours, when it is at a minimum. I cannot understand how night work could explain the unusual irregularity in both this and Dr. Downing's case. A chowkidar's (night watchman) cccupation involves no great physical strain. It merely consists in staying awake at night to watch the sahib's bungalow and sleeping throughout the day-an occupation quite insufficient, I think, inversely to upset the temperature chart during not only health, but also disease."

Of the cases which came under my care during the past eighteen months, that reported here is the only one in which an inverse type of temperature occurred, and the patient was the only one in the series whose work was nocturnal.

Mary F. Lacey. 